\title{
Comparison of short-term clinical outcomes between robot- assisted minimally invasive esophagectomy and video-assisted minimally invasive esophagectomy: a systematic review and meta-analysis
}

\author{
Chao Zheng ${ }^{1 *}$, Xiao-Kun Li ${ }^{1 \#}$, Chi Zhang ${ }^{2 \#}$, Hai Zhou ${ }^{3}$, Sai-Guang Ji ${ }^{3}$, Ji-Hong Zhong ${ }^{2}$, Yang $\mathrm{Xu}^{4}$, \\ Zhuang-Zhuang Cong ${ }^{2}$, Gao-Ming Wang ${ }^{5}$, Wen-Jie Wu ${ }^{6}$, Yi Shen ${ }^{1,2,3}$ \\ ${ }^{1}$ Department of Cardiothoracic Surgery, Jinling Hospital, School of Medicine, Southeast University, Nanjing, China; ${ }^{2}$ Department of Cardiothoracic \\ Surgery, Jinling Hospital, Medical School of Nanjing University, Nanjing, China; ${ }^{3}$ Department of Cardiothoracic Surgery, Nanjing Second Hospital, \\ Medical School of Southeast University, Nanjing, China; ${ }^{4}$ Department of Cardiothoracic Surgery, Jinling Hospital, School of Clinical Medicine, \\ Nanjing Medical University, Nanjing, China; ${ }^{5}$ Department of Thoracic Surgery, Xuzhou Central Hospital, Xuzhou School of Clinical Medicine of \\ Nanjing Medical University, Nanjing, China; ${ }^{6}$ Department of Clinical Medicine, School of Medicine, Southeast University, Nanjing, China \\ Contributions: (I) Conception and design: C Zheng, XK Li, C Zhang, Y Shen; (II) Administrative support: JH Zhong, GM Wang, Y Shen; (III) \\ Collection and assembly of data: H Zhou, SG Ji, JH Zhong, Y Xu, ZZ Cong; (IV) Data analysis and interpretation: C Zheng, XK Li, C Zhang; (V) \\ Manuscript writing: All authors; (VI) Final approval of manuscript: All authors. \\ \#These authors contributed equally to this work. \\ Correspondence to: Yi Shen. Department of Cardiothoracic Surgery, Jinling Hospital, School of Medicine, Southeast University, Nanjing, China. \\ Email: dryishen@nju.edu.cn.
}

\begin{abstract}
Background: Though robot-assisted minimally invasive esophagectomy (RAMIE) is demonstrated to offer a better visualization and provide a fine dissection of the mediastinal structures to facilitate the complex thoracoscopic operation, the superiorities of RAMIE over MIE have not been well verified. The aim of this study was to explore the actual superiorities through comparing short-term results of RAMIE with that of MIE.

Methods: PubMed, EMBASE and web of science databases were systematically searched up to September 1, 2020 for case-controlled studies that compared RAMIE with TLMIE.

Results: Fourteen studies were identified, with a total of 2,887 patients diagnosed with esophageal cancer, including 1,435 patients subjected to RAMIE group and 1,452 patients subjected to MIE group. The operative time in RAMIE was still significantly longer than that in MIE group (OR $=0.785$; 95\% CI, 0.6180.952; $\mathrm{P}<0.001)$. The incidence of pneumonia was significantly lower in RAMIE group compared with MIE group (OR $=0.677 ; 95 \%$ CI, 0.468-0.979; $\mathrm{P}=0.038$ ).

Conclusions: RAMIE has the superiorities over MIE in short-term outcomes in terms of pneumonia and vocal cord palsy. Therefore, RAMIE could be considered as a standard treatment for patients with esophageal cancer.
\end{abstract}

Keywords: Robot-assisted minimally invasive esophagectomy (RAMIE); video-assisted minimally invasive esophagectomy; short-term clinical outcomes; meta-analysis

Submitted Sep 15, 2020. Accepted for publication Nov 03, 2020.

doi: $10.21037 /$ jtd-20-2896

View this article at: http://dx.doi.org/10.21037/jtd-20-2896

$\wedge$ ORCID: 0000-0002-5286-6485. 


\section{Introduction}

Esophageal cancer poses a serious global health burden with high morbidity and mortality rates $(1,2)$. The longterm prognosis of esophageal cancer remains poor with a 5-year overall survival rate of about $15-34 \%(3,4)$. Radical esophagectomy with lymphadenectomy is still the primary treatment for patients with esophageal cancer (5). Conventional open thoracotomy for esophagectomy was found to have considerable morbidity and mortality rates (6). Minimally invasive esophagectomy (MIE), with less invasiveness compared to open thoracotomy, has become increasingly prevalent in the treatment of resectable esophageal cancer since the early 2000s (7). Recently, robotassisted minimally invasive esophagectomy (RAMIE) has also been introduced, which showed significant superiorities of increased magnification, three-dimensional visual clarity, and dexterity (8-10). It filters out the tremor of the surgeon, restores the natural hand-eye coordination axis as a result of the ergonomically designed surgeon's console, and offers more degrees of freedom through its articulating surgical instruments $(9,11)$. Although RAMIE is demonstrated to offer better visualization and enable meticulous dissection of the mediastinum structures facilitating the complex thoracoscopic procedures, the actual superiorities of RAMIE over MIE have not been well verified (10). Several retrospective studies have found that RAMIE was equivalent or even superior to MIE in radical lymphadenectomy. There was only one meta-analysis that compares RAMIE to MIE in 2019 (12). This meta-analysis includes 8 case control studies and no randomized controlled trials. It concluded that RAMIE and MIE display similar effects and safety in the treatment of esophageal cancer. RAMIE could reduce the risk of recurrent laryngeal nerve damage due to improved vision and flexibility during the paratracheal lymph node dissection. However, more high-quality studies have been published until now. The aim of this study was to investigate the actual advantages of RAMIE over MIE by comparing short-term outcomes of RAMIE with that of MIE. We present the following article in accordance with the PRISMA reporting checklist (available at http://dx.doi. org/10.21037/jtd-20-2896).

\section{Methods}

PubMed, EMBASE, and web of science were searched from 1980 to September 1, 2020. Medical subject heading (MeSH) and Thesaurus were used in PubMed and OVID, respectively. According the PICOs, the keywords were determined and identical in the two databases (Medline and EMBASE): "Esophag*", "Esophag* Neoplasm", "Esophag* Cancer*", "Esophag* resection", "Robot*". The PICO format was adopted to establish specific selection criteria in which $\mathrm{P}$ was referred to patients undergoing MIE, I was referred to RAMIE, $\mathrm{C}$ was referred to thoraco-laparoscopic MIE (TLMIE), O was referred to the short-term clinical outcomes. The design style was limited to case-control studies. Only articles published in English met the inclusion criteria. In this meta-analysis, short-term clinical outcomes were classified into infectious complications, including pneumonia, anastomotic leakage, empyema and surgical site infection, and surgical relevant complications, composed of chylothorax and vocal cord palsy, as well as mortality and the length of hospital and ICU stay. The following studies were excluded: narrative or expert reviews, experimental data such as animal studies or trials, unable to acquire primary data and essential information from authors, articles published not in English. The following patients were excluded: patients lost for follow-up, patients discontinued intervention, patients combined with other cancers, patients did not undergo MIE, patients with major organic dysfunction, patients with severe preoperative infection.

\section{Quality assessment}

Selected studies were all case-control studies thus NOS was used for grading. The risk of bias assessment was carried out by two reviewers independently (Chao Zheng, Chi Zhang). A third reviewer (Xiao-Kun Li) arbitrated unresolved disagreements.

\section{Statistical analysis}

Stata 15.1 was applied in data analyses. Forest plots were conducted to show the pooled estimate for the studies. The continuous outcome variables were presented through weighted mean difference (WMD) with 95\% confidence interval (CI), if the same unit and magnitude were applied in all the included researches; otherwise, standard mean difference (SMD) would be applied. Dichotomous outcomes were evaluated using odds ratio (OR) with $95 \%$ CI. In studies that only reported medians and ranges, the mean and standard deviation (SD) were assessed using the means of the method provided by Hozo et al. (13). Heterogeneity was evaluated by $\chi^{2}$ based test and $\mathrm{p}$ value. 


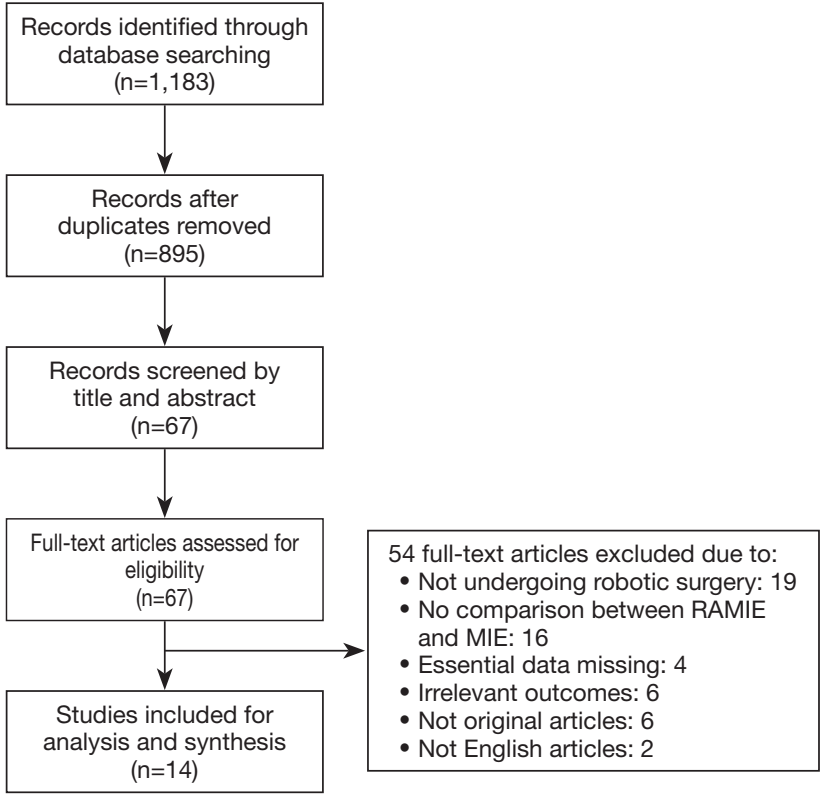

Figure 1 Flow chart of selection for included studies.

If small heterogeneity was observed $\left(\mathrm{I}^{2}<50 \%\right.$ or $\left.\mathrm{P}>0.05\right)$, the fixed-effect model was used; if not, the random-effect model would be used. Sensitivity analysis would be used to investigate the source of heterogeneity. Publication bias was assessed using funnel plot, and Egger's test or Begg's test would be conducted if needed. A P value of less than 0.05 was considered as statistically significant.

\section{Results}

\section{Basic characteristics}

Literature research and selection in this meta-analysis were shown in Figure 1. A total of 1,183 studies were systematically searched from the three electronic databases, after removing 288 duplicates. After reviewing titles and abstracts, 828 studies were removed due to not fulfilling the inclusion criteria. The full-text of remaining 67 studies were further screened. Finally, 14 studies $(9,10,14-25)$ were included finally, with a total of 2,887 patients, including 1,435 patients subjected to RAMIE group and 1,452 patients subjected to TLMIE group. All the included studies reported that the thoracic procedure of esophagectomy was via thoracic approach rather than cervical approach. The reconstruction of the esophagus and gastric conduit was done through cervical anastomosis in most researches. The basic characteristics of included researches are presented in
Table 1. Seven out of 14 studies were carried out in China. All studies were of high-quality score based on NOS. The characteristics and methodological quality assessment scores of the included studies are summarized in Table 2.

\section{Meta-analysis}

Operative time was reported in 11 studies including 1,270 participates. The data was deemed to be heterogeneity $\left(\chi^{2}=165.04, \mathrm{P}<0.0001, \mathrm{I}^{2}=93.9 \%\right)$. Random-effect model was adopted. The operative time in RAMIE was significantly longer than that in MIE group (WMD $=30.929 ; 95 \%$ CI, 4.357-57.500; $\mathrm{P}=0.023)$. To find out the source of large heterogeneity, we did a sensitivity analysis and exclude the results conducted by Yang et al. (23) and Chen et al. (22) to make the $\mathrm{I}^{2}$ to $74.4 \%$. The pooled results were recalculated through a random effect model, and the operative time in RAMIE was still significantly longer than that in MIE group $(W M D=45.497 ; 95 \% C I, 25.900-65.093 ; \mathrm{P}<0.001)$ (Figure 2). The postoperative complications were reported in six studies enrolling 1,021 patients. Heterogeneity was identified $\left(\chi^{2}=2.38, \mathrm{P}=0.79, \mathrm{I}^{2}=0.0 \%\right)$, and fixed effect model was used. No significant difference was found between two groups $(\mathrm{OR}=1.24 ; 95 \% \mathrm{CI}, 0.951-1.617$; $\mathrm{P}=0.11)$. Eleven studies reported data regarding the incidence of anastomotic leak which enrolled 1352 patients. Heterogeneity was not observed $\left(\chi^{2}=10.10, \mathrm{P}=0.432\right.$, $\left.\mathrm{I}^{2}=1.0 \%\right)$, and the forest plot was done using fixed-effect model and no significant difference was observed between RAMIE group and MIE group (OR $=1.127$; 95\% CI, 0.781$1.625 ; \mathrm{P}=0.52)$. Egger test was used to assess publication bias and no publication bias was found in the meta-analysis of the incidence of anastomotic leak $(\mathrm{P}=0.058)$ (Figure 3). We furtherly conducted a sub-analysis due to different approaches of anastomosis, and the result indicated that no significant difference was observed between two groups no matter cervical anastomosis or thoracic anastomosis were performed (Figure S1). Chylothorax was reported in six researches consisting of 518 patients. Heterogeneity was identified $\left(\chi^{2}=0.61, \mathrm{P}=0.99, \mathrm{I}^{2}=0.0 \%\right)$, and fixed-effect model was used. No significant difference was found in the incidence of chylothorax between two groups $(\mathrm{OR}=0.41$; 95\% CI, 0.125-1.32; $\mathrm{P}=0.13)$. Nine studies including 1,191 patients reported the incidence of pneumonia. Statistical heterogeneity was not significant $\left(\chi^{2}=3.55, \mathrm{P}=0.90, \mathrm{I}^{2}=0.0 \%\right)$. The incidence of pneumonia in RAMIE group was significantly lower than that in MIE group (OR $=0.677 ; 95 \%$ CI, 0.468-0.979; $\mathrm{P}=0.038)$. The incidence of surgical site 
Table 1 Characteristics of the selected studies included in the meta-analysis

\begin{tabular}{|c|c|c|c|c|c|c|c|c|c|c|}
\hline Study & Country & Group & $\mathrm{N}$ & Age, years & $\mathrm{BMI}, \mathrm{kg} / \mathrm{m}^{2}$ & $\begin{array}{l}\text { Site (upper/ } \\
\text { mid/lower) }\end{array}$ & $\begin{array}{l}\text { Pathology } \\
\text { (ESCC/EAC) }\end{array}$ & $\begin{array}{l}\text { Neoadjuvant } \\
\text { therapy }\end{array}$ & Tumor size $(\mathrm{cm})$ & $\begin{array}{l}\text { Surgical } \\
\text { procedures }\end{array}$ \\
\hline \multirow[t]{2}{*}{ Suda 2012} & Japan & RAMIE & 16 & $65[53-86]$ & $\begin{array}{l}21.3 \\
(17.5-26.3)\end{array}$ & $2 / 7 / 9$ & $16 / 0$ & 6 & $3.75[0.9-8.0]$ & NA \\
\hline & & MIE & 20 & 64.5 [50-79] & $\begin{array}{l}20.4 \\
(14.9-24.8)\end{array}$ & $2 / 12 / 6$ & $20 / 0$ & 17 & $3.65[0.0-7.0]$ & NA \\
\hline $\begin{array}{l}\text { Weksler } \\
2012\end{array}$ & & MIE & 26 & $64.3 \pm 11.3$ & 27.9 & NA & $3 / 23$ & 10 & NA & Ivor-Lewis \\
\hline \multirow{2}{*}{$\begin{array}{l}\text { Yerokun } \\
2016\end{array}$} & Canada & RAMIE & 170 & $56 / 64 / 70$ & NA & 0/156/14 & NA & 120 & 20.0/35.0/50.0 & NA \\
\hline & & MIE & 170 & $56 / 63 / 69$ & NA & $0 / 160 / 10$ & NA & 120 & $22.5 / 35.0 / 50.0$ & NA \\
\hline Park 2016 & & MIE & 43 & $64.3 \pm 8.0$ & $23.3 \pm 3.1$ & $7 / 9 / 27$ & $43 / 0$ & 4 & NA & Mckown \\
\hline \multirow{2}{*}{$\begin{array}{l}\text { Weksler } \\
2017\end{array}$} & American & RAMIE & 569 & $62.9 \pm 9.6$ & NA & NA/513/NA & $114 / 455$ & 405 & 33.7 [17-57] & NA \\
\hline & & MIE & 569 & $62.8 \pm 9.3$ & NA & NA/527/NA & $114 / 455$ & 401 & 35.0 [15-55] & NA \\
\hline \multirow[t]{2}{*}{ Chao 2018} & China & RAMIE & 34 & $56.76 \pm 8.39$ & NA & $10 / 15 / 9$ & $34 / 0$ & 17 & NA & Mckown \\
\hline & & MIE & 34 & $53.47 \pm 8.69$ & NA & $10 / 19 / 9$ & $34 / 0$ & 17 & NA & Mckown \\
\hline \multirow[t]{2}{*}{ Deng 2018} & China & RAMIE & 42 & $60.7 \pm 6.9$ & NA & NA & $42 / 0$ & NA & NA & Mckown \\
\hline & & MIE & 42 & $61.8 \pm 9.5$ & NA & NA & $42 / 0$ & NA & NA & Mckown \\
\hline Chen 2019 & & MIE & 54 & $61.8 \pm 8.3$ & $23.0 \pm 2.7$ & NA & $54 / 0$ & NA & NA & Mckown \\
\hline \multirow{2}{*}{$\begin{array}{l}\text { Motoyama } \\
2019\end{array}$} & Japan & RAMIE & 21 & $63[44-76]$ & NA & $6 / 7 / 8$ & $21 / 0$ & 12 & NA & NA \\
\hline & & MIE & 38 & 66 [49-75] & NA & $9 / 16 / 13$ & $38 / 0$ & 19 & NA & NA \\
\hline \multirow{2}{*}{$\begin{array}{l}\text { Tagkolos } \\
2019\end{array}$} & Germany & RAMIE & 50 & 62 & 26.13 & NA & NA & NA & NA & Ivor-Lewis \\
\hline & & MIE & 50 & 64 & 24.89 & NA & NA & NA & NA & Ivor-Lewis \\
\hline \multirow[t]{2}{*}{ Yang 2019} & China & RAMIE & 271 & $63.4 \pm 7.1$ & NA & $38 / 169 / 64$ & $271 / 0$ & 29 & NA & Mckown \\
\hline & & MIE & 271 & $63.5 \pm 7.4$ & NA & $31 / 171 / 69$ & $271 / 0$ & 28 & NA & Mckown \\
\hline \multirow[t]{2}{*}{ Zhang 2019} & China & RAMIE & 66 & $62.3 \pm 7.8$ & $22.9 \pm 3.1$ & $0 / 29 / 37$ & $64 / 2$ & NA & $3.1 \pm 1.4$ & Ivor-Lewis \\
\hline & & MIE & 66 & $62.0 \pm 7.8$ & $23.1 \pm 4.5$ & $0 / 26 / 40$ & $65 / 1$ & NA & $3.0 \pm 1.4$ & Ivor-Lewis \\
\hline
\end{tabular}


Table 2 The Newcastle-Ottawa scale

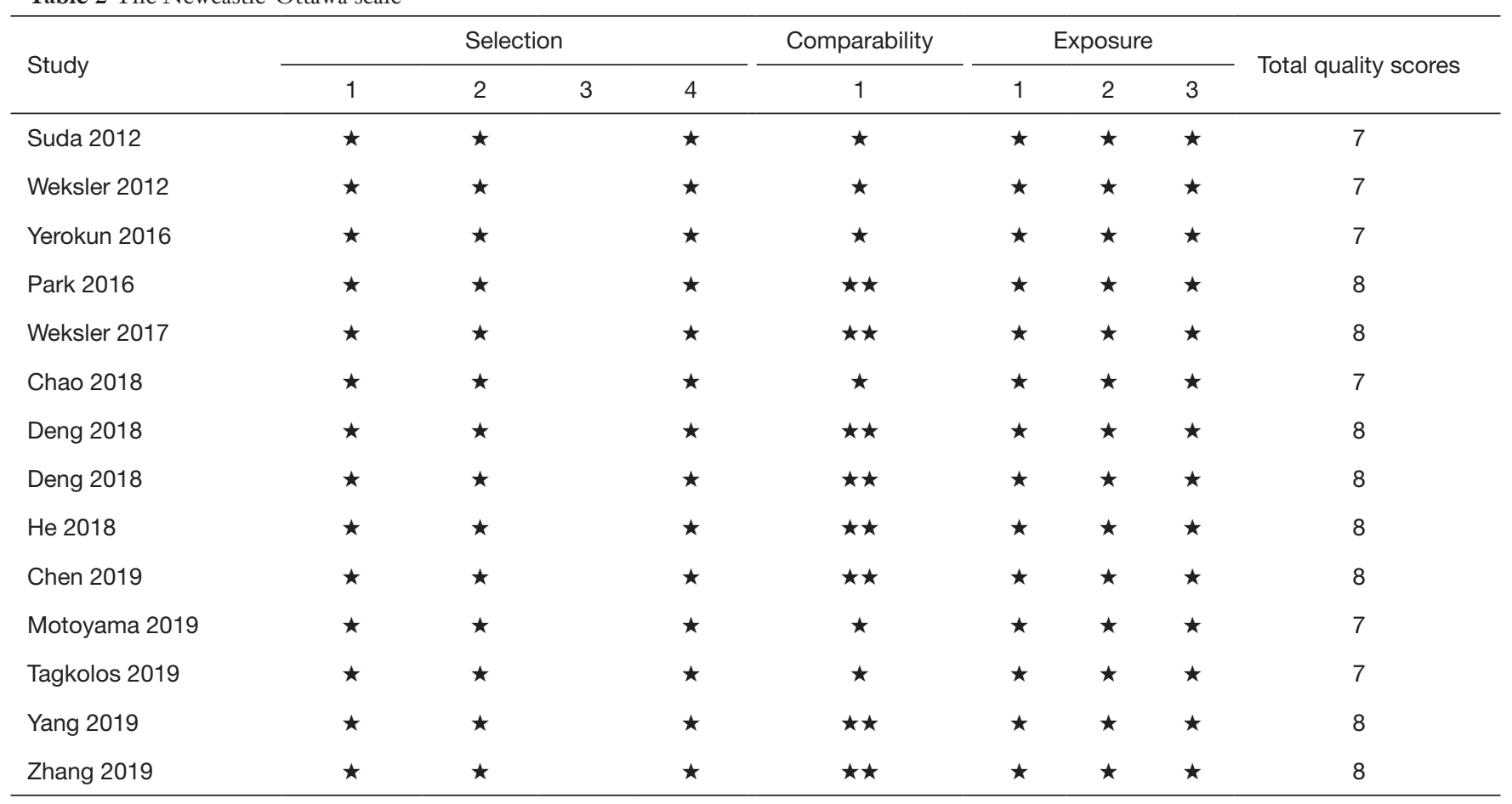

infection was reported in 4 studies including 791 patients. Heterogeneity was identified $\left(\chi^{2}=2.14, \mathrm{P}=0.54, \mathrm{I}^{2}=0.0 \%\right)$. Fixed effect model was used and there was no significant difference between two groups ( $\mathrm{OR}=1.42 ; 95 \% \mathrm{CI}$, $0.412-4.882 ; \mathrm{P}=0.58$ ) (Figure 4). Ten studies reported vocal cord paralysis, including 1,270 patients. The significant heterogeneity $\left(\chi^{2}=27.97, \mathrm{P}=0.001, \mathrm{I}^{2}=67.28 \%\right)$ was observed, then random-effect model was used. There was no significant difference between RAMIE group and MIE group $(\mathrm{OR}=0.833 ; 95 \% \mathrm{CI}, 0.439-1.581 ; \mathrm{P}=0.576)$. To find out the source of large heterogeneity, we did a sensitivity analysis and excluded the results conducted by Yang et al. to make the $\mathrm{I}^{2}$ to $19.6 \%$. The pooled results were recalculated through a fixed-effect model, the incidence of vocal cord palsy was significantly different between two groups (OR =0.624; 95\% CI, 0.411-0.947; $\mathrm{P}=0.027$ ) (Figure 5). 30 days mortality was reported in 7 studies including 1,947 patients. Heterogeneity was identified $\left(\chi^{2}=2.83, \mathrm{P}=0.587\right.$, $\left.\mathrm{I}^{2}=0.0 \%\right)$. Fixed effect model was used and no significant difference was found between RAMIE group and MIE group $(\mathrm{OR}=1.678 ; 95 \% \mathrm{CI}, 0.986-2.857$; $\mathrm{P}=0.056)$. 90 days mortality was also reported in 7 studies including 2,165 participates. Heterogeneity was identified $\left(\chi^{2}=10.06\right.$, $\left.\mathrm{P}=0.790, \mathrm{I}^{2}=0.0 \%\right)$ and fixed effect model was used. No significant difference was seen between two groups (OR
=1.106; 95\% CI, 0.754-1.621; P=0.777) (Figure 6).

\section{Discussion}

According to the only randomized controlled trial so far, MIE was found to be superior to open esophagectomy in terms of intraoperative blood loss, acute immunological response, postoperative pulmonary infections, length of hospital stay, postoperative pain scores, and quality of life (7). Although MIE seems to improve short-term clinical outcomes, it is a highly complex procedure to master due to the combination of two-dimensional vision, mirrored intracorporeal movements of the instruments, moving target anatomy, and nearby vital structures that need to be avoided $(7,8,25,26)$. Therefore, RAMIE was introduced to aid in overcoming the technical limitations of conventional MIE, showing significant superiority of increased magnification, three-dimensional visual clarity, and dexterity (24,27). A ROBOT trial was conducted, which compared RAMIE to open esophagectomy regarding postoperative morbidity, mortality, and survival (28). A total of 112 patients were randomized in a high-volume tertiary referral center that was experienced in both techniques (28). The overall complication rate was significantly lower in the RAMIE group, which was likely attributable to significantly 


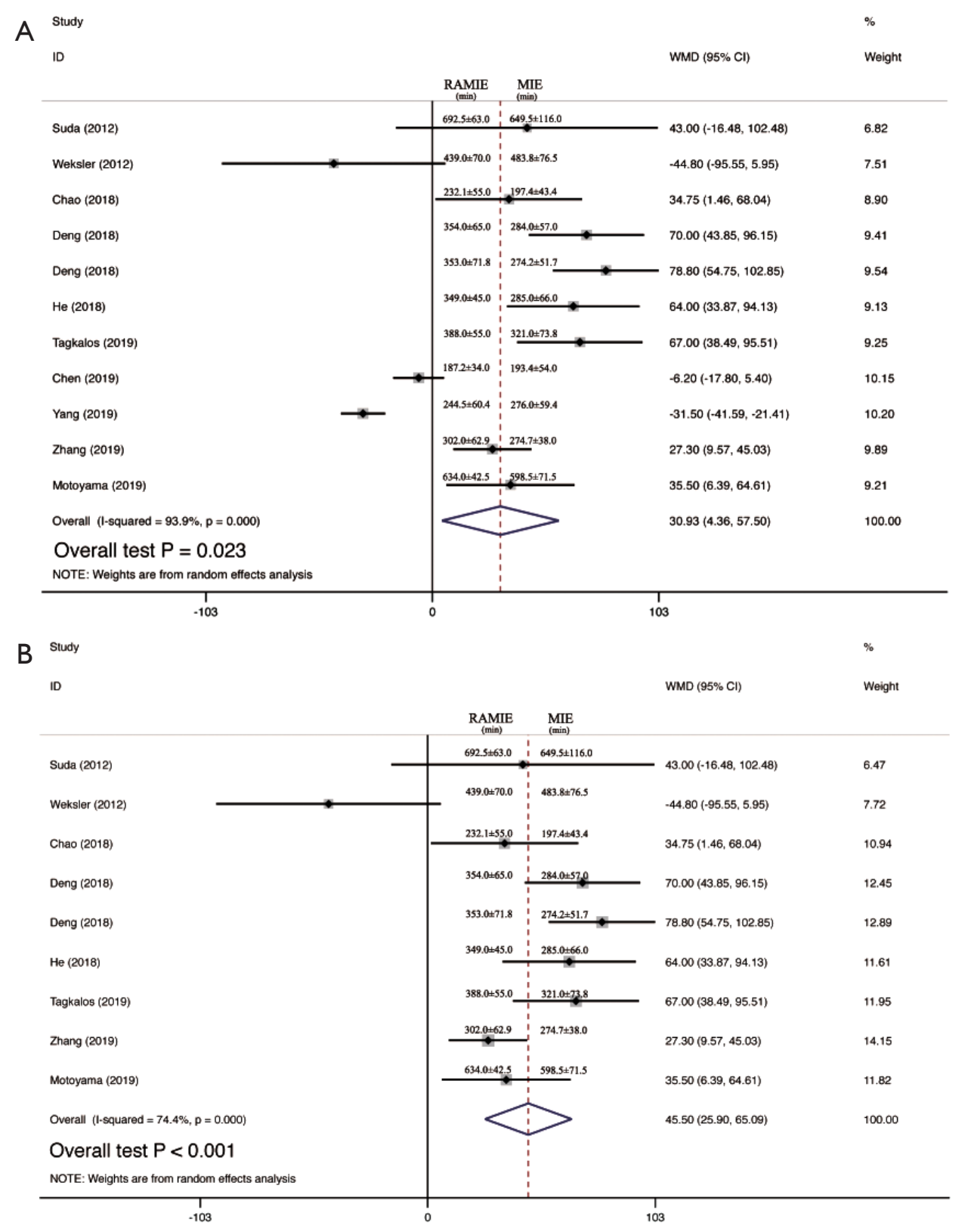

Figure 2 Comparison of operative time between RAMIE and MIE (A) and after excluding two studies (B). RAMIE, robot-assisted minimally invasive esophagectomy; MIE, minimally invasive esophagectomy.

lower rates of cardiac complications (47\% vs. $22 \%$ ) and pulmonary complications (32\% vs. 58\%) (29). Furthermore, RAMIE was associated with less intraoperative blood loss, lower postoperative pain scores, faster functional recovery, and better quality of life when compared to open esophagectomy. Whether RAMIE has any superiorities over MIE on short-term clinical outcomes remains controversial.
There was only one meta-analysis reported by Jin et al. (12), including 8 studies, comparing the short-term clinical outcomes between RAMIE and the conventional MIE. There were no significant differences between RAMIE and MIE in terms of postoperative complications, length of hospital stays or mortality. We conducted this metaanalysis, enrolling 14 high-quality retrospective studies, to 

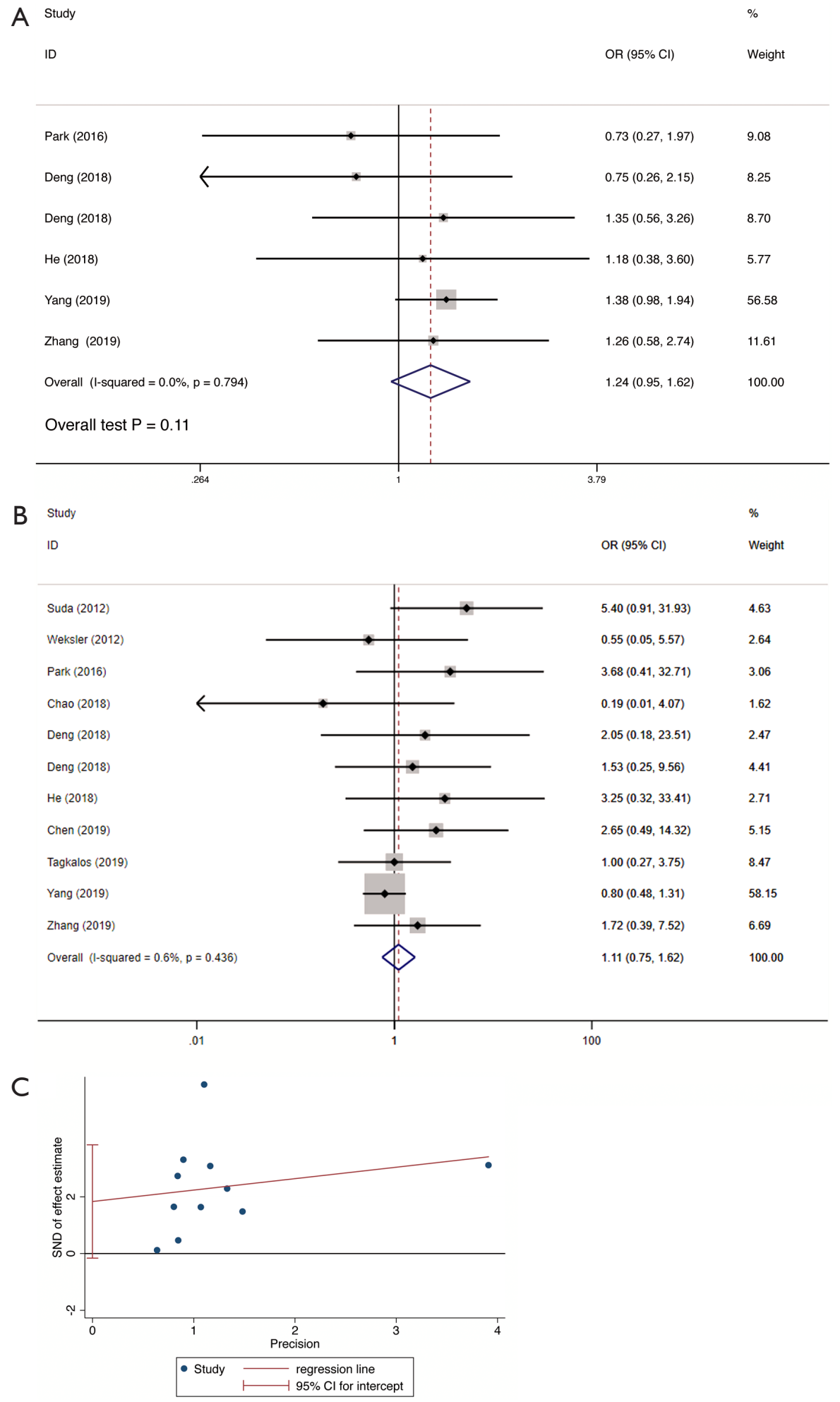

Figure 3 Comparison of the incidence of postoperative complications (A) and anastomotic leak (B) with responding Eger's test (C) between RAMIE and MIE. RAMIE, robot-assisted minimally invasive esophagectomy; MIE, minimally invasive esophagectomy. 


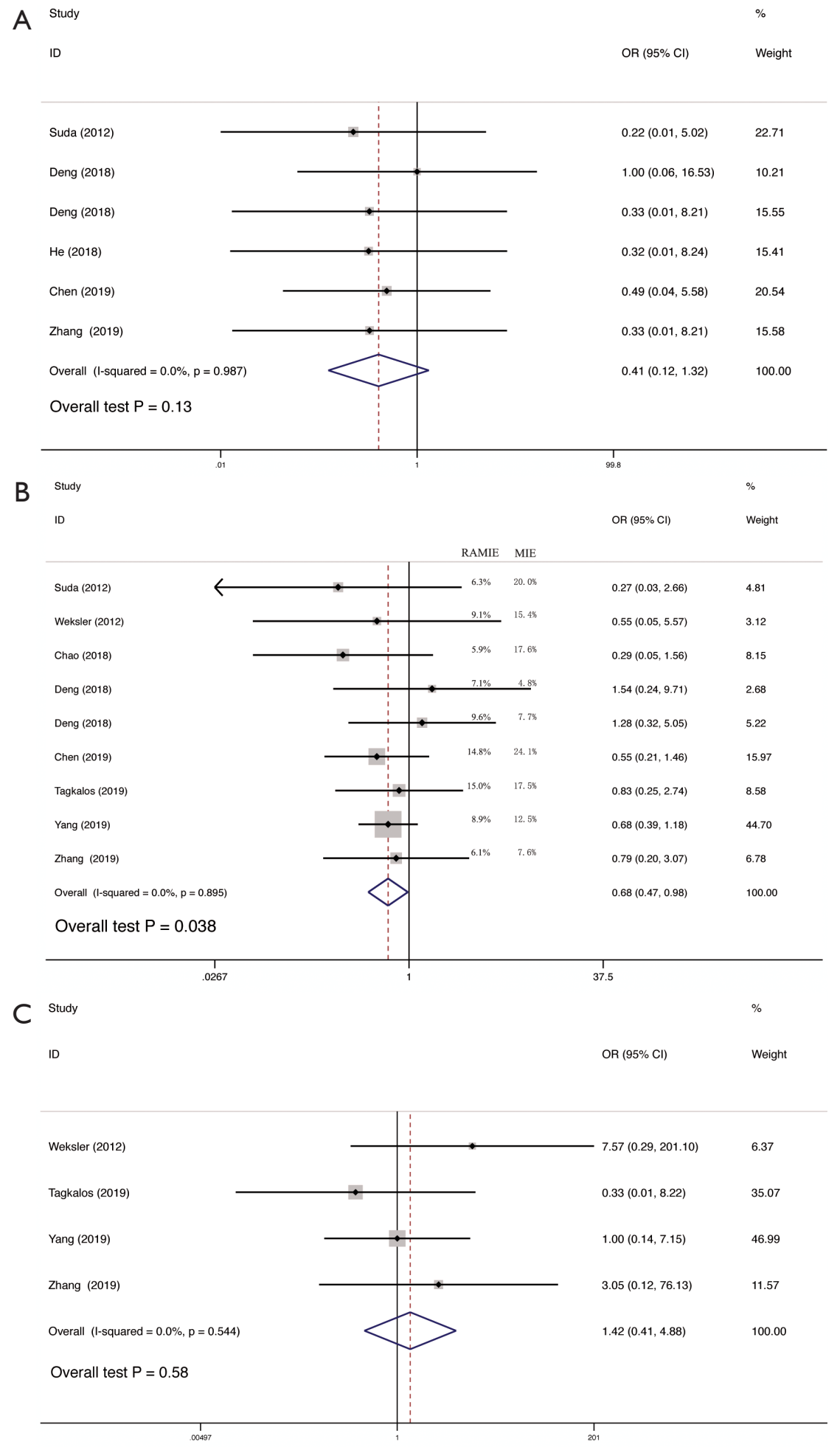

Figure 4 Comparison of the incidence of chylothorax (A), pneumonia (B), and surgical site infection (C) between RAMIE and MIE. RAMIE, robot-assisted minimally invasive esophagectomy; MIE, minimally invasive esophagectomy. 


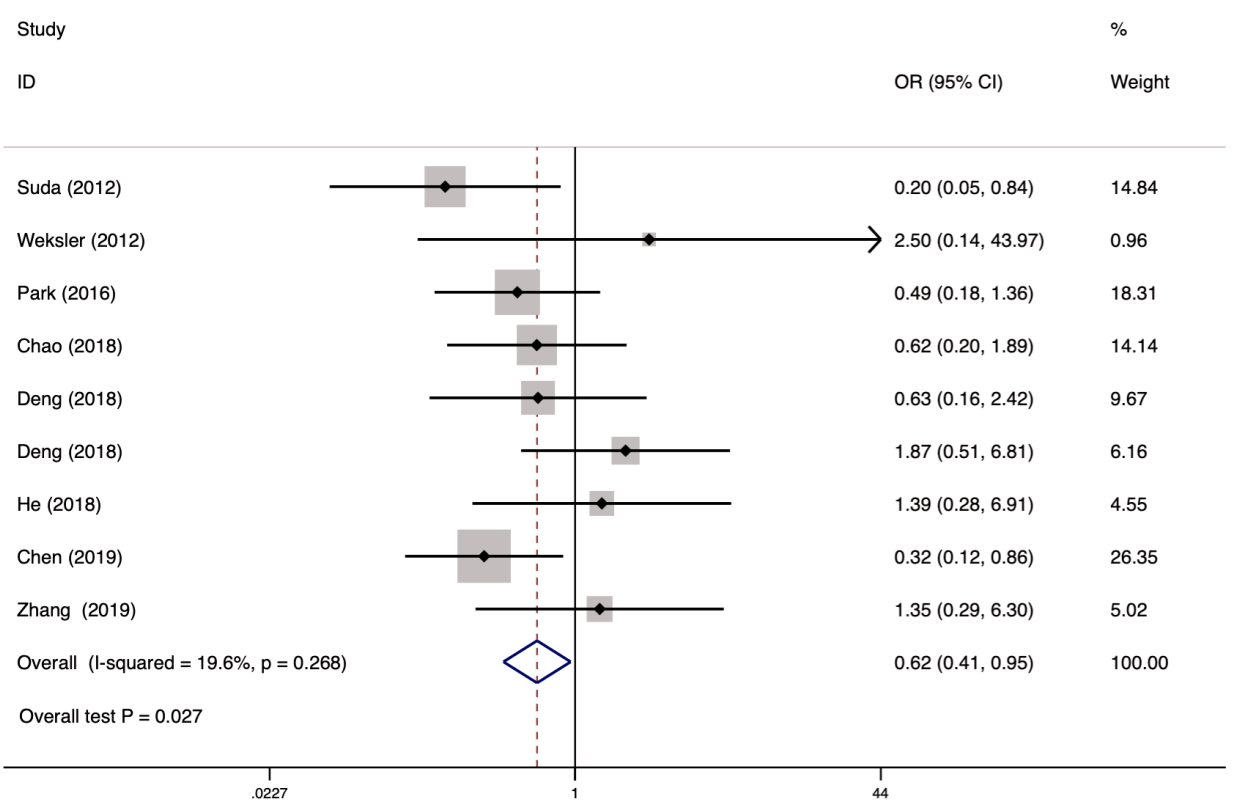

Figure 5 Comparison of the incidence of vocal cord palsy between RAMIE and MIE. RAMIE, robot-assisted minimally invasive esophagectomy; MIE, minimally invasive esophagectomy.

further evaluate the short-term clinical outcomes in RAMIE by comparing with that in MIE.

According to the results, the incidences of pneumonia and vocal cord palsy were significantly lower in RAMIE group than that in MIE group. The potential reasons why RAMIE has the superiorities over MIE could be explained as followed: (I) RAMIE provided a three-dimensional visualization with ten-times-enlarged image and superior imaging quality facilitating identifying various structures during operations. (II) RAMIE provided with freely articulated movement of the robotic arms and seven-degree freedom of its dexterity endowrists which make surgeons operate in a stable and comfortable environment, enabling more precise dissection and avoiding nerve injury especially in vocal cord palsy as well as other structures injury during lymphadenectomy. The incidence of anastomotic leak in RAMIE group was tended to be higher than that in MIE group. This may be because of the induced ischemia of the stump caused by too much exposure of the cranial end of the divided esophagus which could be due to the robotic system providing a better surgical view and more accurate dissection than the standard thoracoscopic approach. There was no significant difference between two groups in 30-day mortality and 90-day mortality which at least demonstrated that RAMIE was equivalent to MIE in terms of safety. However, the operative time in RAMIE was significantly longer than that in MIE. The potential reasons may be:
(I) the robotic carts need to be repositioned between the thoracic phase and the abdominal phase which result in RAMIE needing to install twice in each operation; (II) surgeons were more familiar with MIE since they have performed MIE for many years while only conducted RAMIE for a few years. With the development of robotic technique and surgeons' proficiency, the operative time would be shortened according to the learning curve. The operative time in RAMIE group in the study of Yang et al. reported in 2019 was significantly longer than that in MIE group $(\mathrm{P}<0.001)(23)$. Meanwhile, in the study of Chen et al. reported in 2019, the mean operative time in RAMIE was also longer than that in MIE, however, there was no significant difference (22).

Four studies reported long-term outcomes. The difference of 5 -year overall survival (69\% vs. $59 \%$ for RAMIE and MIE, respectively, $\mathrm{P}=0.737$ ) demonstrated in the study of Park et al. (15) was not statistically significant. Similarly, Weksler et al. (17) reported that the median survival time between two groups (48 vs. 49 months for RAMIE and MIE, respectively, $\mathrm{P}>0.05$ ) was not significantly different. Besides, both Yerokun et al. (16) and Yang et al. (23) demonstrated that no significant difference among RAMIE group and MIE group was observed in terms of 3-year overall survival. In addition, Yang et al. (23) also reported 3-year disease-free survival (DFS) and the results indicated that there was no statistical difference in DFS between 
A study

ID
OR $(95 \% \mathrm{Cl})$

Weight

\begin{tabular}{|c|c|c|c|}
\hline Babantude (2016) & & $1.00(0.20,5.03)$ & 13.65 \\
\hline Park (2016) & & $2.12(0.08,53.33)$ & 2.66 \\
\hline Deng (2018) & & $0.33(0.01,8.22)$ & 6.87 \\
\hline Tagkolos (2019) & & $0.33(0.01,8.22)$ & 6.86 \\
\hline Weksler (2017) & & $2.06(1.12,3.80)$ & 69.95 \\
\hline Chao (2018) & & (Excluded) & 0.00 \\
\hline Zhang (2019) & & (Excluded) & 0.00 \\
\hline Overall (I-squared $=0.0 \%, p=0.587$ ) & & $1.68(0.99,2.86)$ & 100.00 \\
\hline $\begin{array}{c}1 \\
.0129\end{array}$ & 1 & $\begin{array}{c}1 \\
77.8\end{array}$ & \\
\hline Study & & & $\%$ \\
\hline ID & & OR $(95 \% \mathrm{Cl})$ & Weight \\
\hline
\end{tabular}

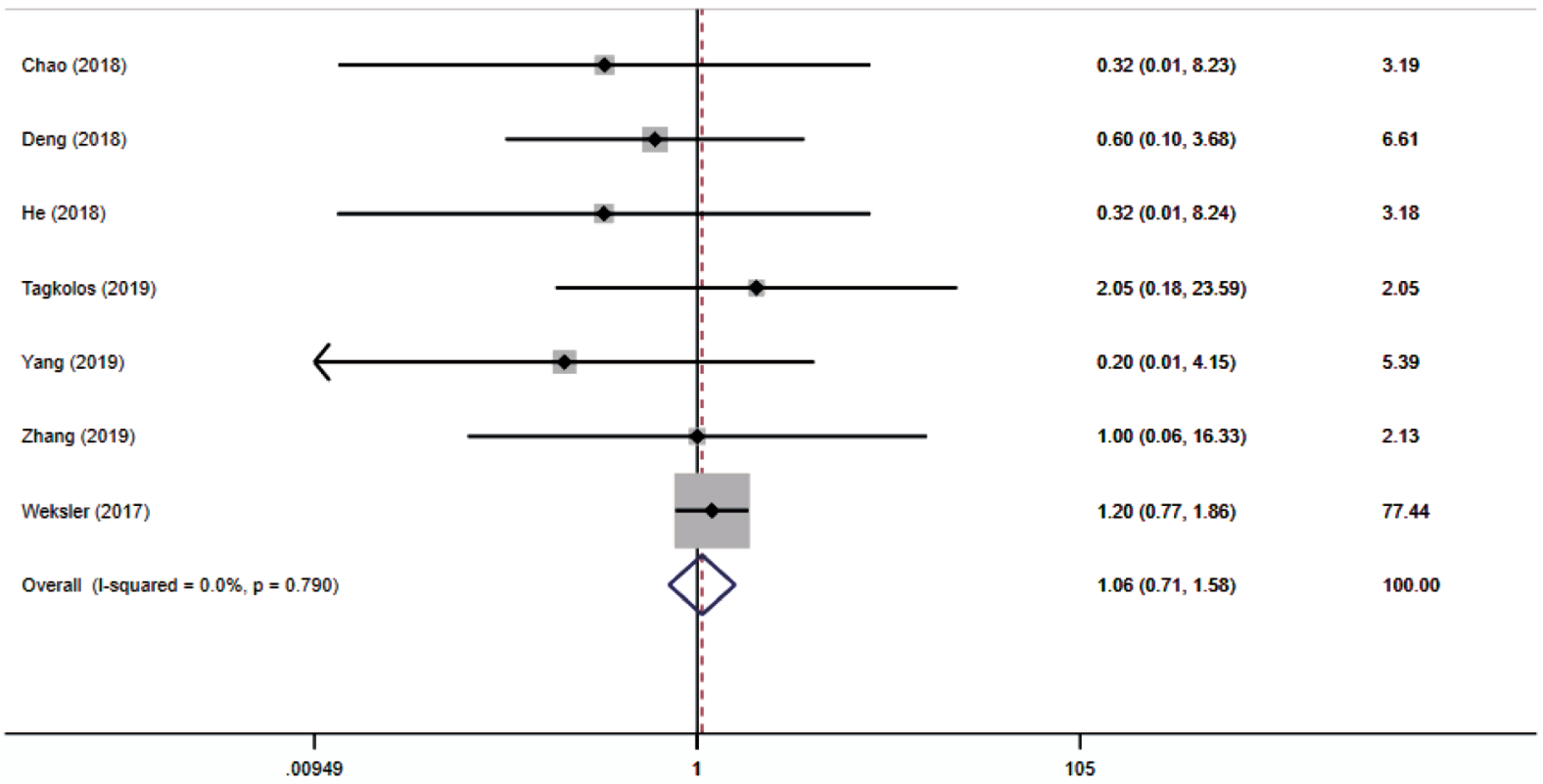

Figure 6 Comparison of the 30-day (A) and 90-day mortality (B) between RAMIE and MIE. RAMIE, robot-assisted minimally invasive esophagectomy; MIE, minimally invasive esophagectomy. 
RAMIE and MIE groups.

Despite several studies used PSM analysis, they still cannot rule out the presence of potential selection biases due to the retrospective nature of these studies, meanwhile, longer follow-up is needed to compare the long-term outcomes between two groups. Therefore, RCTs with long-term follow-up were urgently warranted to compare these two approaches. Yang et al. launched a prospectively RCT (RAMIE trial, ClinicalTrial.gov: NCT03094351) to compare RAMIE and MIE in March 2017 (11). Chao et al. also conducted a prospectively RCT registered on 22 October 2018 (ClinicalTrial.gov: NCT03094351) to compare the two techniques (30).

\section{Conclusions}

RAMIE has better short-term outcomes than MIE in terms of pneumonia and vocal cord palsy. However, there were no significant differences in overall and disease-free survival as well as short-term mortality between the two groups. Therefore, RAMIE could be considered as a standard treatment for patients with esophageal cancer.

\section{Acknowledgments}

We thank all the members of the department of cardiothoracic surgery in our hospital that participated in this research. We also thank Xiao-Kun Li for his advice on statistical analysis.

Funding: This work was supported by the National Natural Science Foundation of China (81172032), the Natural Science Foundation of Jiangsu Province (BK20181239) and the Science and Technology Program of Xu Zhou (KCl8037).

\section{Footnote}

Reporting Checklist: The authors have completed the PRISMA reporting checklist. Available at http://dx.doi. org/10.21037/jtd-20-2896

Conflicts of Interest: All authors have completed the ICMJE uniform disclosure form (available at http://dx.doi. org/10.21037/jtd-20-2896). The authors have no conflicts of interest to declare.

Ethical Statement: The authors are accountable for all aspects of the work in ensuring that questions related to the accuracy or integrity of any part of the work are appropriately investigated and resolved.

Open Access Statement: This is an Open Access article distributed in accordance with the Creative Commons Attribution-NonCommercial-NoDerivs 4.0 International License (CC BY-NC-ND 4.0), which permits the noncommercial replication and distribution of the article with the strict proviso that no changes or edits are made and the original work is properly cited (including links to both the formal publication through the relevant DOI and the license). See: https://creativecommons.org/licenses/by-nc-nd/4.0/.

\section{References}

1. Barbour AP, Cormack OMM, Baker PJ, et al. Long-term Health-related Quality of Life Following Esophagectomy: A Nonrandomized Comparison of Thoracoscopically Assisted and Open Surgery. Ann Surg 2017;265:1158-65.

2. Chen W, Zheng R, Baade PD, et al. Cancer statistics in China, 2015. CA Cancer J Clin 2016;66:115-32.

3. Berry MF. Esophageal Cancer: Improvements in Treatment, Staging, and Now Prognostic Indicators? Semin Thorac Cardiovasc Surg 2016;28:559-60.

4. Bray F, Ferlay J, Soerjomataram I, et al. A. Global cancer statistics 2018: GLOBOCAN estimates of incidence and mortality worldwide for 36 cancers in 185 countries. CA Cancer J Clin 2018;68:394-424.

5. Chen MF, Yang YH, Lai CH, et al. Outcome of patients with esophageal cancer: a nationwide analysis. Ann Surg Oncol 2013;20:3023-30.

6. Lv L, Hu W, Ren Y, et al. Minimally invasive esophagectomy versus open esophagectomy for esophageal cancer: a meta-analysis. Onco Targets Ther 2016;9:6751-62.

7. Mariette C, Piessen G, Triboulet JP. Therapeutic strategies in oesophageal carcinoma: role of surgery and other modalities. Lancet Oncol 2007;8:545-53.

8. Ruurda JP, van der Sluis PC, van der Horst S, et al. Robotassisted minimally invasive esophagectomy for esophageal cancer: A systematic review. J Surg Oncol 2015;112:257-65.

9. Suda K, Ishida Y, Kawamura Y, et al. Robot-assisted thoracoscopic lymphadenectomy along the left recurrent laryngeal nerve for esophageal squamous cell carcinoma in the prone position: technical report and short-term outcomes. World J Surg 2012;36:1608-16.

10. Tagkalos E, Goense L, Hoppe-Lotichius M, et al. Robot-assisted minimally invasive esophagectomy (RAMIE) compared to conventional minimally invasive esophagectomy (MIE) for esophageal cancer: a propensitymatched analysis. Dis Esophagus 2020;33:doz060. 
11. Yang Y, Zhang X, Li B, et al. Robot-assisted esophagectomy (RAE) versus conventional minimally invasive esophagectomy (MIE) for resectable esophageal squamous cell carcinoma: protocol for a multicenter prospective randomized controlled trial (RAMIE trial, robot-assisted minimally invasive Esophagectomy). BMC Cancer 2019;19:608.

12. Jin D, Yao L, Yu J, et al. Robotic-assisted minimally invasive esophagectomy versus the conventional minimally invasive one: A meta-analysis and systematic review. Int J Med Robot 2019;15:e1988.

13. Hozo SP, Djulbegovic B, Hozo I. Estimating the mean and variance from the median, range, and the size of a sample. BMC Med Res Methodol 2005;5:13.

14. Weksler B, Sharma P, Moudgill N, et al. Robot-assisted minimally invasive esophagectomy is equivalent to thoracoscopic minimally invasive esophagectomy. Dis Esophagus 2012;25:403-9.

15. Park S, Hwang Y, Lee HJ, et al. Comparison of robotassisted esophagectomy and thoracoscopic esophagectomy in esophageal squamous cell carcinoma. J Thorac Dis 2016;8:2853-61.

16. Yerokun BA, Sun Z, Yang CJ, et al. Minimally Invasive Versus Open Esophagectomy for Esophageal Cancer: A Population-Based Analysis. Ann Thorac Surg 2016;102:416-23.

17. Weksler B, Sullivan JL. Survival After Esophagectomy: A Propensity-Matched Study of Different Surgical Approaches. Ann Thorac Surg 2017;104:1138-46.

18. Chao YK, Hsieh MJ, Liu YH, et al. Lymph Node Evaluation in Robot-Assisted Versus Video-Assisted Thoracoscopic Esophagectomy for Esophageal Squamous Cell Carcinoma: A Propensity-Matched Analysis. World J Surg 2018;42:590-8.

19. Deng HY, Huang WX, Li G, et al. Comparison of shortterm outcomes be-tween robot-assisted minimally invasive esophagectomy and video-assisted minimally invasive esophagectomy in treating middle thoracic esophageal cancer. Dis Esophagus 2018;31:doy012.

20. Deng HY, Luo J, Li SX, et al. Does robot-assisted minimally invasive esophagectomy really have the advantage of lymphadenectomy over video-assisted minimally invasive esophagectomy in treating esophageal squamous cell carcinoma? A propensity score-matched analysis based on short-term outcomes. Dis Esophagus 2019;32:doy110.

21. He H, Wu Q, Wang Z, et al. Short-term outcomes of robot-assisted minimally invasive esophagectomy for esophageal cancer: a propensity score matched analysis. J Cardiothorac Surg 2018;13:52.
22. Chen J, Liu Q, Zhang X, et al. Comparisons of short-term outcomes between robot-assisted and thoraco-laparoscopic esophagectomy with extended two-field lymph node dissection for resectable thoracic esophageal squamous cell carcinoma. J Thorac Dis 2019;11:3874-80.

23. Yang Y, Zhang X, Li B, et al. Short- and mid-term outcomes of robotic versus thoraco-laparoscopic McKeown esophagectomy for squamous cell esophageal cancer: a propensity scorematched study. Dis Esophagus 2020;33:doz080.

24. Zhang Y, Han Y, Gan Q, et al. Early Outcomes of RobotAssisted Versus Thoracoscopic-Assisted Ivor Lewis Esophagectomy for Esophageal Cancer: A Propensity Score-Matched Study. Ann Surg Oncol 2019;26:1284-91.

25. Motoyama S, Sato Y, Wakita A, et al. Extensive Lymph Node Dissection Around the Left Laryngeal Nerve Achieved With Robot-assisted Thoracoscopic Esophagectomy. Anticancer Res 2019;39:1337-42.

26. Sato Y, Kosugi S, Aizawa N, et al. Risk Factors and Clinical Outcomes of Recurrent Laryngeal Nerve Paralysis After Esophagectomy for Thoracic Esophageal Carcinoma. World J Surg 2016;40:129-36.

27. Yun JK, Lee IS, Gong CS, et al. Clinical utility of robotassisted transthoracic esophagectomy in advanced esophageal cancer after neoadjuvant chemoradiation therapy. J Thorac Dis 2019;11:2913-23.

28. van der Sluis PC, Ruurda JP, van der Horst S, et al. Robot-assisted minimally invasive thoraco-laparoscopic esophagectomy versus open transthoracic esophagectomy for resectable esophageal cancer, a randomized controlled trial (ROBOT trial). Trials 2012;13:230.

29. van der Sluis PC, van der Horst S, May AM, et al. Robot-assisted Minimally Invasive Thoracolaparoscopic Esophagectomy Versus Open Transthoracic Esophagectomy for Resectable Esophageal Cancer: A Randomized Controlled Trial. Ann Surg 2019;269:621-30.

30. Chao YK, Li ZG, Wen YW, et al. Robotic-assisted Esophagectomy vs Video-Assisted Thoracoscopic Esophagectomy (REVATE): study protocol for a randomized controlled trial. Trials 2019;20:346.

Cite this article as: Zheng C, Li XK, Zhang C, Zhou H, Ji SG, Zhong JH, Xu Y, Cong ZZ, Wang GM, Wu WJ, Shen Y. Comparison of short-term clinical outcomes between robotassisted minimally invasive esophagectomy and video-assisted minimally invasive esophagectomy: a systematic review and meta-analysis. J Thorac Dis 2021;13(2):708-719. doi: 10.21037/ jtd-20-2896 
Supplementary

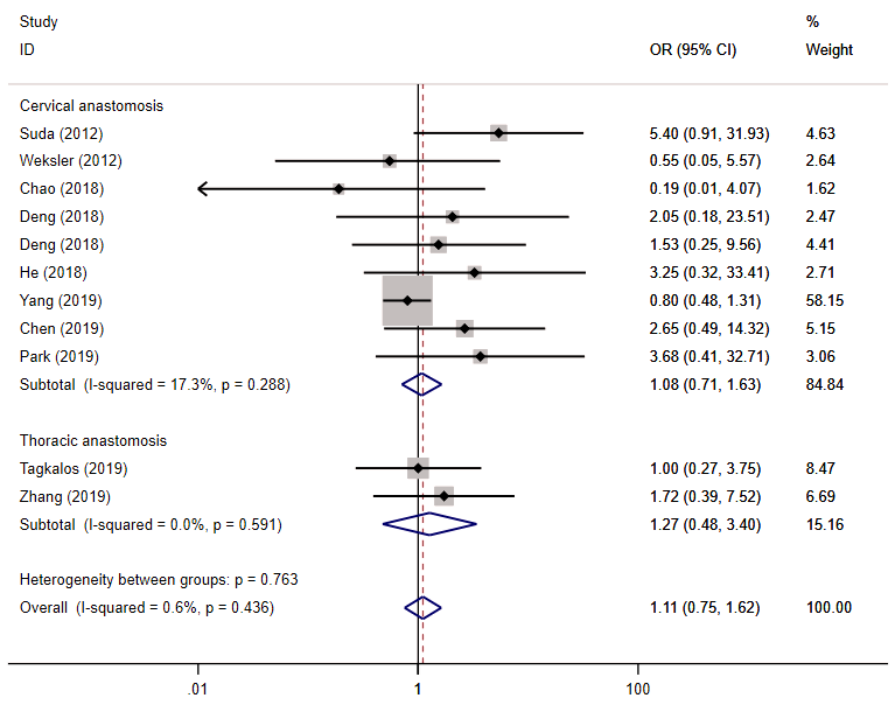

Figure S1 Comparison of the incidence of anastomotic leakage stratified by cervical or thoracic anastomosis. 